\title{
EXPERIMENTAL AND NUMERICAL STUDIES OF TURBULENT FLOW IN AN IN-LINE TUBE BUNDLES
}

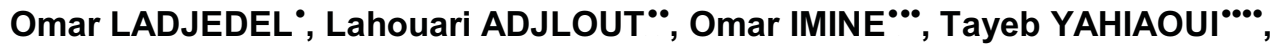 \\ Mohamed AOUNALAH
}

\begin{abstract}
In the present paper an experimental and a numerical simulation of the turbulent flow in an in-line tube bundles have been performed. The experiments were carried out using a subsonic wind tunnel. The pressure distributions along the tubes (22 circumferential pressure taping) were determined for a variation of the azimuthal angle from 0 to 360deg. The drag and lift forces are measured using the TE 44 balance. The Navier-Stokes equations of the turbulent flow are solved using Reynolds Stress and $K-\varepsilon$, turbulence models (RANS) provided by Fluent CFD code. An adapted grid using static pressure, pressure coefficient and velocity gradient, furthermore, a second order upwind scheme were used. The obtained results from the experimental and numerical studies show a satisfactory agreement.
\end{abstract}

\section{INTRODUCTION}

The heat transfer and nuclear power generation industrial sector is showing much interest in the experimental investigation and numerical simulation of cross-flow in tube bundle arrays. Much like single cylinders the tube response inside tube bundles is also highly prone to oscillations. The turbulence and periodic excitation by coherent vorticity phenomenon may lead to severe vibrations thus causing extensive damage to tube bundles over a short period of time.

Sumner et al. [1] have done an extensive experimental study on two and three cylinders placed side by side for a Reynolds number range of 500-3,000 the transverse ratio $(T / D)$ is varied from 1.0 to 6.0. It has been observed that both the two cylinder and three cylinder configurations show various flow patterns for different gap spaces. In the intermediate region which is also the interest region of our study an asymmetrical flow pattern is reported. It is also observed by Kim \& Durbin [2] that the biased flow pattern

\footnotetext{
- Laboratoire d'aérohydrodynamique naval Département de génie maritime, mechanical engineering faculty, USTO Oran 31000 Algeria (e-mail: ladjedelomar@yahoo.com).

- Laboratoire d'aerohydrodynamique naval département de genie maritime, mechanical engineering faculty, usto oran 31000 algeria (e-mail: adjloutl@yahoo.fr)

-.-Laboratoire d'aéronautique et systèmes propulsive, Département de Génie Mécanique, Mechanical Engineering Faculty, USTO Oran, 31000 Algeria, (e-mail: imine_omar@yahoo.fr)

.... Laboratoire d'aeronautique et systemes propulsive, departement de genie mecanique, mechanical engineering faculty, usto oran, 31000 algeria, (e-mail:yahiaoui_tayeb@yahoo.fr)

.... laboratoire d'aérohydrodynamique naval Département de génie maritime, mechanical engineering faculty, USTO Oran 31000 Algeria (e-mail: aounallah_2000@yahoo.fr)
} 
switches intermittently from being directed towards one cylinder to the other. They concluded that this flow behavior is independent of Reynolds number and purely dependent upon the $T / D$ ratio. Summer et al. [1] further conclude that the flow deflection decreases as the $T / D$ ratio is Increased. Wolfe and Zaida [3] used a feedback control on vortex shedding from two tandem cylinders. It was concluded that when a cylinder is placed in the wake of another cylinder then its unsteady loading is not only dependent upon the flow behavior in its own wake but also on the flow pattern in the wake of the upstream cylinder. Feenstra et al. [4] experimentally study flow-induced vibrations in a cantilevered tube bundle array with single and two phase cross-flow. For a single phase flow the study addresses two cases; a single flexible tube in an otherwise rigid tube bundle and a fully flexible tube bundle configuration. It was observed that for the single flexible tube configuration fluid elastic instability was achieved at $25 \%$ higher flow velocity and symmetric vortex shedding occurred at $50 \%$ higher flow velocities. The paper of Feenstra et al. [4] presents an excellent comparison with previous experimental studies conducted by Paidoussis [5] and Weaver\& Fitzpatrick [6]at a gap ratio of 1.5 using a water tunnel. Traub [7] conducts open wind tunnel experiments to study the influence of turbulence intensity on pressure drop in in-line and staggered tube bundles at various Reynolds numbers. It is observed that the drag coefficient remains more or less the same for a wide range of Reynolds numbers and only changes slightly for very high Reynolds numbers. It is also concluded from this study that as the Reynolds number becomes very high the recirculation region shrinks due to shifting of the point of flow separation. Due to this shifting, the pressure drop decreases and hence the drag coefficient decreases. At a low Reynolds number of 200 Lam et al. [8] performed a particle image velocimetry on a four cylinder square array where the gap ratio was 4.Lam and Fang [9] perform an experimental study on the effect of gap ratio on the flow over a square four cylinder in-line configuration. The paper discusses flow pattern, pressure distribution and lift and drag forces on cylinders at a Reynolds number of 12,800 based on free stream velocity. It is seen that at small gap ratios due to the suppression of the wake region vortex shedding is hardly formed. Moreover for these gap ratios the stagnation point is not at zero degrees rather the shift is 20-50 degrees from flow direction. Benhamadouche and Laurence [10] discuss cross flow in a staggered tube bundle array with different levels of grid refinement. It has been stated both by this study and by Rollet-Miet et al. [11] that standard 2 equations RANS models fail to produce reliable results for flow inside tube bundles. Rollet-Miet et al. [11] suggest that for LES there is little difference in results between the Smagorinsky and the Dynamic Smagorinsky models. Based on this conclusion Benhamadouche and Laurence [10] used a Smagorinsky model, a centered convection scheme and the SIMPLEC method for pressure and velocity coupling. The Reynolds number based on bulk velocity was 9,000, whereas the $Y+$ for the fine LES mesh was less than 7. It is stated that the flow occasionally switches direction and can take a 45degree orientation, a phenomenon which also frequently appears in in-line tube bundle configurations. Findings report that the high levels of fluctuations are due to oscillations of the flow around cylinder.

DNS of similar tube bundles is Moulinec et al. [12] in which a staggered array is tested with $P / D$ variation from 2 to 3 . The cases are tested for very low Reynolds number (less than 6,000$)$. The interesting thing is that the study tries to explain a lot of unanswered questions regarding tube bundles. Questions such as what is the sufficient domain size needed for a numerical simulation? What is the flow transition Reynolds number from 2$D$ to 3-D? What is the effect of gap spacing on flow behavior? In Moulinec et al. the time discretization was performed by Adams-Bashforth scheme and spatial discretization were performed using second order central differencing scheme. It was concluded that in staggered tubes vortex shedding appears at a Reynolds number of 125 as opposed to a single cylinder case where shedding appears at a Reynolds number of 40 . It was also concluded that for the single cylinder case the flow becomes turbulent at Reynolds number of 2000 whereas in this case the Reynolds number was found to be 6,000 . Finally it is found that at for this Reynolds number due to increased turbulent dispersion 
of the wake vorticity, wake disappearance occurs. One such paper worth mentioning is Cheng et al. [13] in which a matrix of in-line cubes have been tested by various numerical simulations including $k-\varepsilon$, Smagorinsky, dynamic Smagorinsky and Localized dynamic model at a Reynolds number of 3,800 based on bulk velocity. The study concludes that qualitative results of all the numerical schemes including $k-\varepsilon$ model compare quite well. However, quantitatively the $k-\varepsilon$ model over predicts the separation region. On the other hand predictions obtained with the Smagorinsky model are off by only a few percent. It is also noted that the use of the Dynamic model leads to additional unphysical backscatter of energy for which various cures have to be thought of in the future. For LES cases spatial discretizations are carried out using second order central differencing scheme and the time discretization using Crank-Nicholson scheme. Finally it was concluded that although complex features like vortex shedding and separation zones are predicted better with LES, the cost of computation increases significantly. Experimental investigation of turbulent flow in an in-line tube bundle were performed in wind tunnel, using flow control by adding grooves to the cylinders and simulated numerically by using the code ANSYS Fluent.

\section{EXPERIMENTAL FACILITY}

The Wind Tunnel shown in figure 1 is of the closed circuit, horizontal return type with a working section $460 \mathrm{~mm}$ high, $460 \mathrm{~mm}$, wide and $1200 \mathrm{~mm}$ long. The Closed Circuit Wind Tunnel is of conventional design and has advantages over a similar open circuit design. These include; a higher maximum velocity, lower power consumption and lower noise level. It is driven by an A.C motor and axial flow fan that forces air around the circuit and produces a maximum velocity of $60 \mathrm{~m} / \mathrm{s}$.

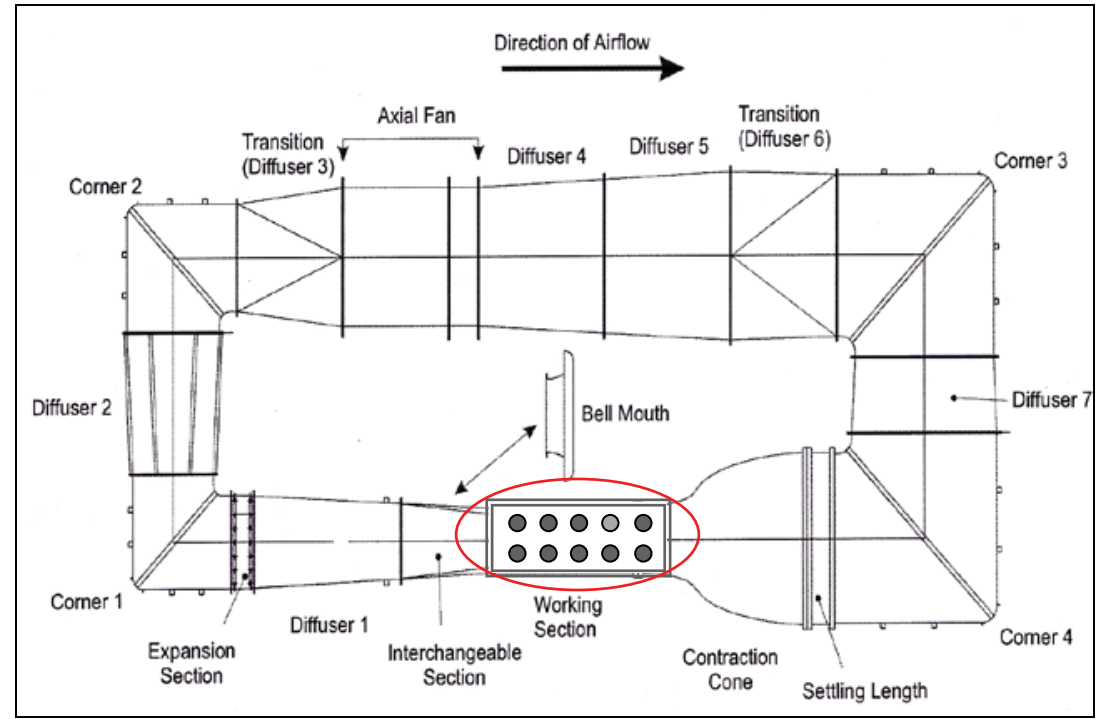

Figure 1: Wind tunnel used fitted with the new working section.

Figure. 2 shows the new working section of the tube bundle model used. The bundle consists of seven rows of PVC tubes of outer diameter of $40 \mathrm{~mm}$ arranged in an online array. As shown in the figure, each row has seven full tubes. Half tubes were also mounted along the bottom and top walls of the test model alternately to simulate an infinite tube bundle and minimize the wall boundary layer. 


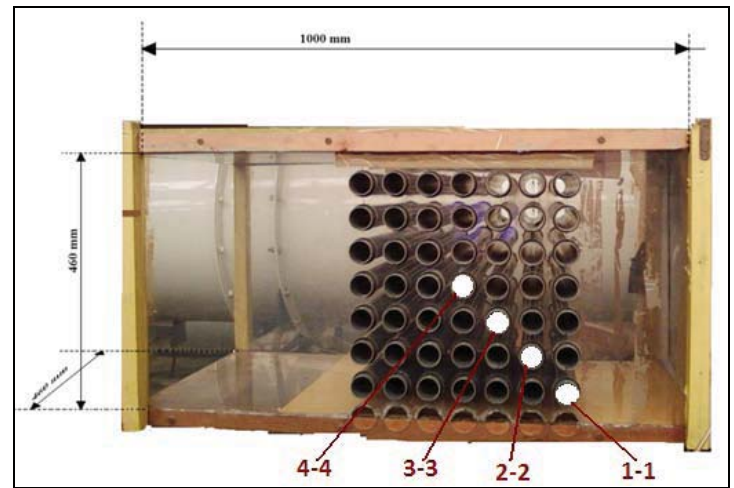

Figure 2: New working section and the geometrical features of the tube bundle.

The pressure measurements were carried out for the 49 tubes as shown in Figure 3.The measurement of pressure distribution around the cylinder for an azimuthal angle from $0^{\circ}$ to $360^{\circ}$ for the circular cylinders with two grooves is carried out. 22 pressures tapings are installed on the cylinder circumference that's making possible to measure pressure distribution in any plan in the working area. The geometrical characteristics of the cylinders used in the bundle are presented in Figure 4 where the form and dimensions of the grooves are also shown. In the present work, the drag forces are measured using the TE81 balance and the pressure distributions on the cylinder were performed using a TE44DPS differential pressure scanner. The latter pressure scanning box allowed sequential selection of up to 20 pressure tapings. The display unit links to a computer, loaded with DATASLIM software for data analysis and logging of results. The experiments setup is shown in figure 3.The uncertainty of the pressure measurement was $0.5 \mathrm{~mm}$ $\mathrm{H}_{2} \mathrm{O}$.

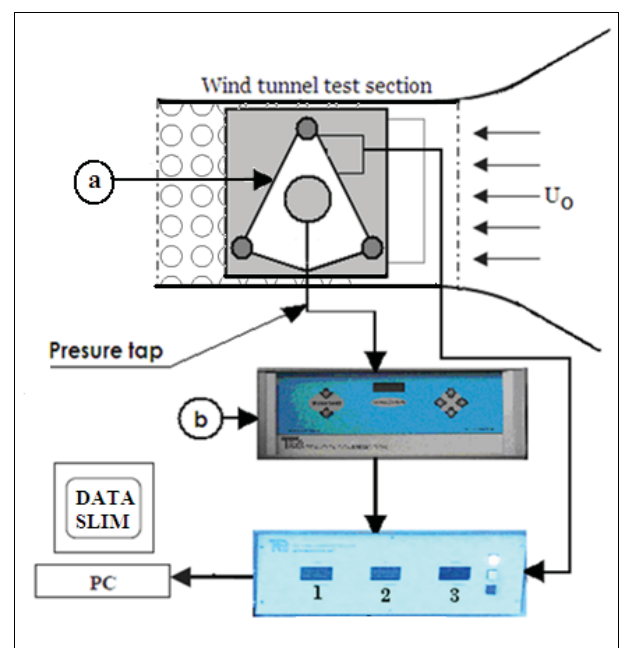

Figure 3: Measurement hardware of drag and pressure.
a: balance TE81
b: TE44 DPS

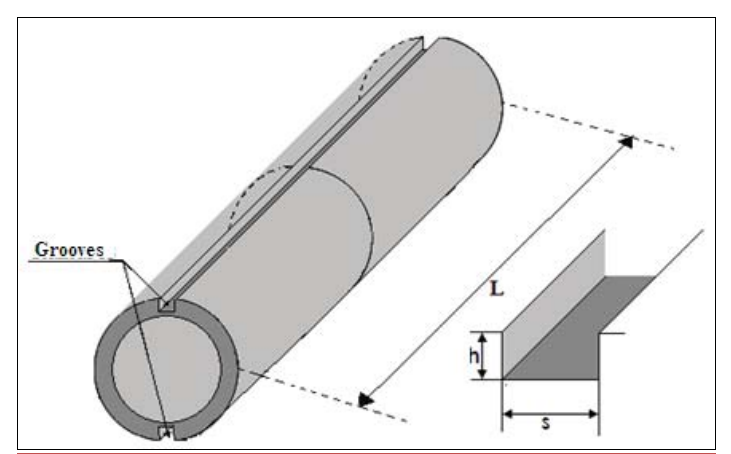

Figure 4 : Form and dimensions of the grooves

$s$ indicates the width of the groove, $h$ is the depth of the groove and $L$ is the length of the groove. 


\section{NUMERICAL STUDY}

Figure 5 shows the geometry of the two-dimensional domain representing a typical region in a bundle, the geometry and the boundary conditions corresponds perfectly remain the same as the experimental work but the grooved cylinders are replaced by smooth cylinders to have a comparison. Fluid with an approach velocity, a velocity profile based on $U_{0}$, enters the solution domain instead of $U_{0}$ uniformly entered at the inlet region, a distance $L_{u s}=40 * D$ from the centre of the first tube. The raison of using a velocity profile is that the location of inlet will not have a significant impact on the solutions obtained and to unburden the domain from additional cells that has a negative effect on the computational cost. The outlet section is placed a distance $L_{o}$ from the centre of the last tube.

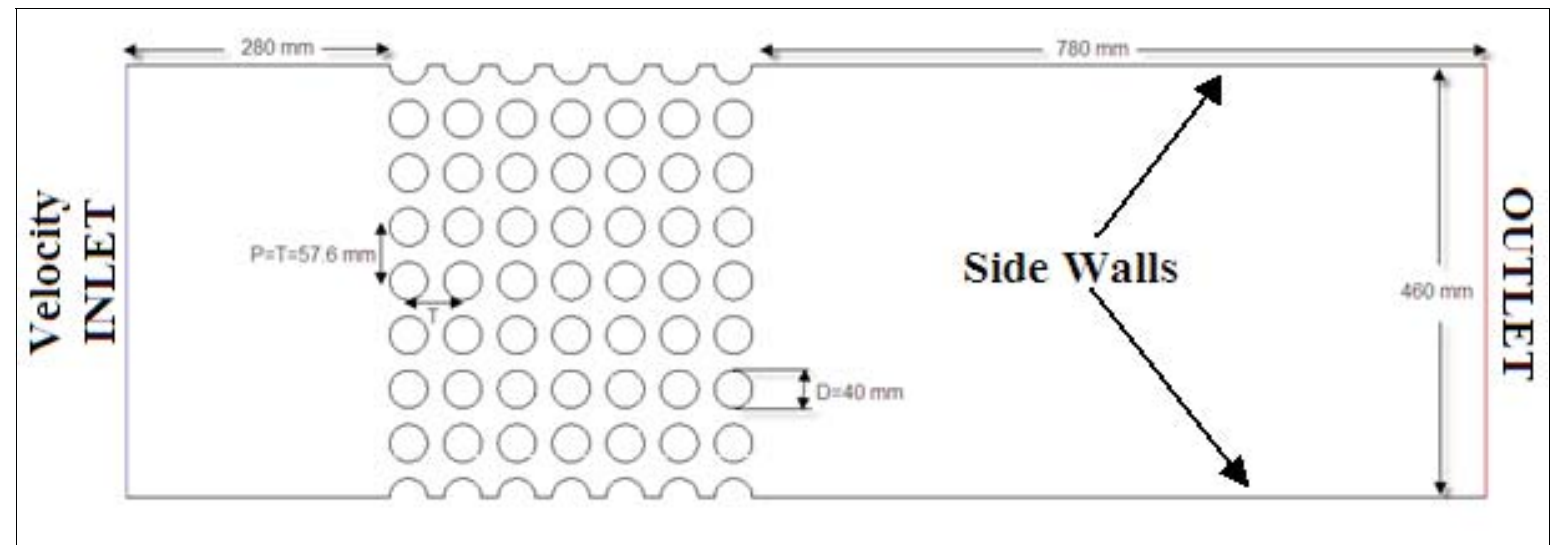

Figure 5: Cross-sectional view of the tube bundle model with the definition of geometrical parameters.

\subsection{Governing Equations}

In the present study a numerical investigation of an across flow in an in-line tube bundles has been performed. The Navier-Stokes equations of the turbulent flow are solved using, $K-\varepsilon$, and Reynolds Stress turbulence models (RSM) provided by Fluent CFD code. The flow is considered to be two-dimensional, incompressible, steady, and turbulent. The fluid is Newtonian with constant density $\rho$ and dynamic viscosity $\mu$ The Reynolds Averaged Navier-Stokes (RANS) equations for continuity and momentum conservation can be written as:

$$
\begin{gathered}
\frac{\partial U_{j}}{\partial x_{j}}=0 \\
\frac{\partial}{\partial x_{j}}\left(\rho U_{j} U_{i}\right)=\frac{\partial}{\partial x_{j}}\left(\mu \frac{\partial U_{i}}{\partial x_{j}}-\partial \overline{u_{j} u_{j}}\right)-\frac{\partial P}{\partial x_{i}}
\end{gathered}
$$

The two turbulence models used in this study are one five equation model; Reynolds Stress two two-equation model and $k-\varepsilon$ (Launder and Spalding 1994.

The first model is Suitable for complex 3D flows with strong streamline curvature; strong swirl/rotation (e.g. curved duct, Rotating flow passages, swirl combustors with very large inlet swirl, cyclones).this model is governed by the exact transport equations for the transport of the Reynolds Stresses, $\rho \overline{u_{i}^{\prime} u_{j}^{\prime}}$ which can be written as follows: 


$$
\begin{aligned}
& \frac{\partial}{\partial t}\left(\rho \overline{u_{i}^{\prime} u_{j}^{\prime}}\right)+\frac{\partial}{\partial x_{k}}\left(\rho u_{k} \overline{u_{i}^{\prime} u_{j}^{\prime}}\right)=-\frac{\rho}{\rho x_{k}}\left[\rho \overline{u_{i}^{\prime} u_{j}^{\prime} u_{k}}+\overline{p\left(\delta_{k j} u_{i}^{\prime}+\delta_{i k} u_{j}^{\prime}\right)}\right] \\
& +\frac{\partial}{\partial x_{k}}\left[\mu \frac{\partial}{\partial x_{k}}\left(\overline{u_{i}^{\prime} u_{j}^{\prime}}\right)\right]-\rho\left(\overline{u_{i}^{\prime} u_{k}^{\prime}} \frac{\partial u_{j}}{\partial x_{k}}+\overline{u_{j}^{\prime} u_{k}^{\prime}} \frac{\partial u_{i}}{\partial x_{k}}\right)-\rho \beta\left(g_{i} \overline{u_{j} \theta}+g_{j} \overline{u_{i} \theta}\right) \\
& +p\left(\frac{\partial u_{i}^{\prime}}{\partial x_{j}}+\frac{\partial u_{j}^{\prime}}{\partial x_{i}}\right)-2 \mu \frac{\partial u_{i}^{\prime}}{\partial x_{k}} \frac{\partial u_{j}^{\prime}}{\partial x_{k}}-2 \rho \Omega_{k}\left(u_{j}^{\prime} u_{m}^{\prime} \epsilon_{i k m}+\overline{u_{i}^{\prime} u_{m}^{\prime}} \epsilon_{i k m}\right)+S_{u s e r}
\end{aligned}
$$

Of the various terms in these exact equations, $C_{i j}, D_{L, i j}, P_{i j}$ and do not require any modeling. However, $D_{t, i j}, G_{i j}, \phi_{i j}$, and $\epsilon_{i j}$ need to be modeled to close the equation.

The second model $k-\varepsilon$ is the most widely used in engineering turbulence model for industrial applications. It has a Robust and reasonably accurate and it has many submodels for compressibility, buoyancy, and combustion.

The $\mathrm{k}-\varepsilon$ model consists of the turbulent kinetic energy $(k)$ equation:

$$
\frac{D}{D t}(\rho k)=\frac{\partial}{\partial x_{j}}\left[\left(\mu+\frac{\mu_{t}}{\sigma_{k}}\right) \frac{\sigma k}{\sigma x_{j}}\right]+G_{k}-\rho \varepsilon
$$

And the dissipation rate $(\varepsilon)$ equation:

$$
\frac{D}{D t}(\rho \varepsilon)=\frac{\partial}{\partial x_{j}}\left[\left(\mu+\frac{\mu_{t}}{\sigma_{\varepsilon}}\right) \frac{\sigma \varepsilon}{\sigma x_{j}}\right]+C_{e l} \frac{\varepsilon}{k} G_{k}-\rho C_{\varepsilon 2} \frac{\varepsilon^{2}}{k}
$$

Where $C_{\mu}=0.09, C_{\varepsilon 1}=1.44, C_{\varepsilon 2}=1.92, \sigma_{k}=1, \sigma_{\varepsilon}=1.3$

Several grids have been tested to investigate the influence of grid refinement on the solution; hence, three meshes were employed for the case of $\operatorname{Re}=35500$. Figure 6 shows the grids used in the simulation. Figure 7 shows the pressure coefficient distributions for the three grids tested by the RSM model. It is clearly seen that there is a little difference between the three grids. The medium grid is used in all subsequent calculations.

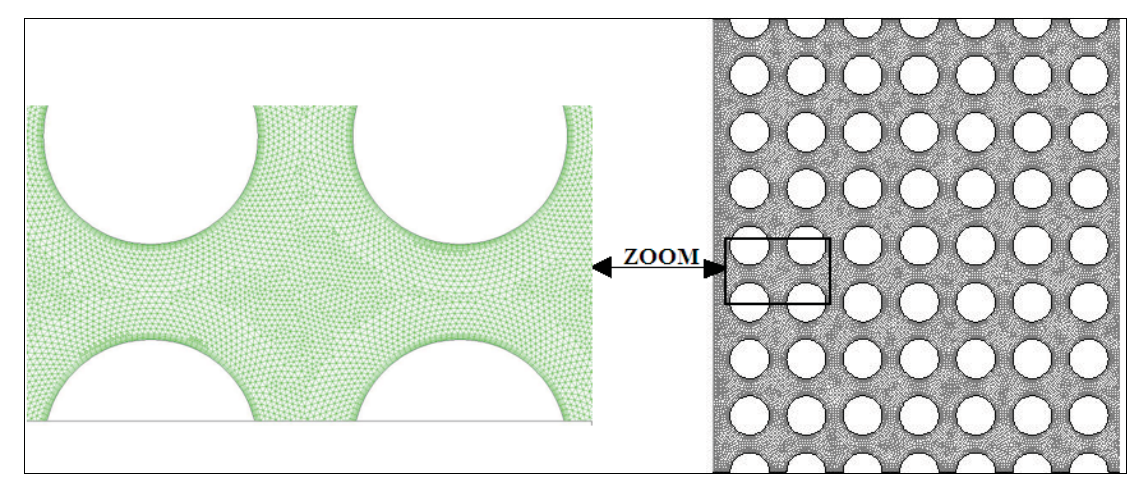

Figure 6: Grid used in the simulation. 


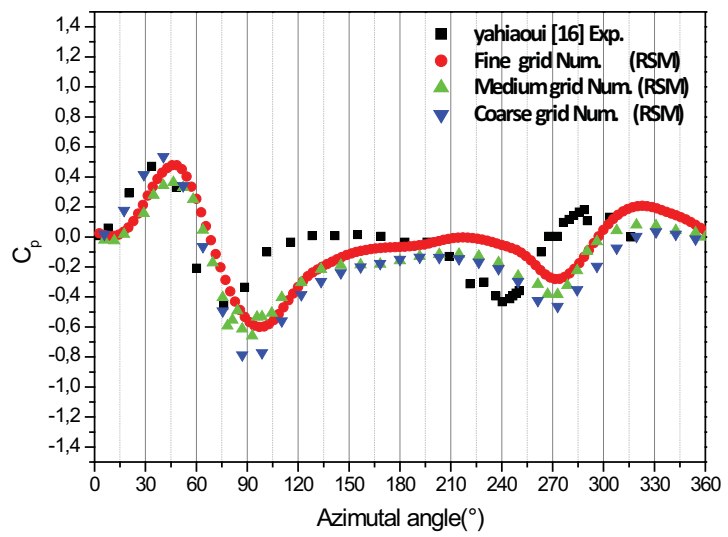

Figure 7: Grids independence test

\section{RESULt AND DISCUSSION}

The experimental and numerical tests were performed for the following physical parameters:

- Reynolds number $=35500$ (based on diameter and gap bulk velocity);

- Free stream and Gap bulk velocity: $U_{0}=3.1321 \mathrm{~m} / \mathrm{s}, U g=10.2505 \mathrm{~m} / \mathrm{s}\left(U_{0}\right.$ Mean flow velocity, Ug gap bulk velocity);

- sampling time $=1$ second;

- pitch ratio, $P / D=1.44(P=T=57.6 \mathrm{~mm}, D=40 \mathrm{~mm})$.

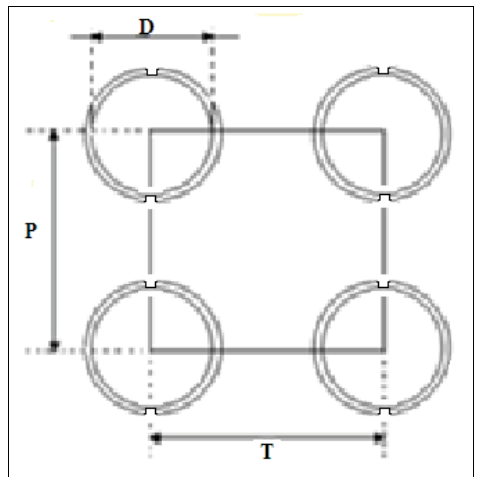

Figure 8: Pitch ratios of tube bundles

The difference in pressure between the surface pressure, $\mathrm{P}$, and the static pressure, $\mathrm{P}_{\text {ref, }}$ is divided by the dynamic pressure to obtain the pressure coefficient, for correlations and the discussion to follow; the coefficient of pressure is defined as:

$$
C_{P}=\frac{p-p_{r e f}}{0.5\left(\rho \cdot U_{g}^{2}\right)}
$$

Where $U g$ is the gap velocity, $U_{\infty}$ is the free stream or the inlet velocity, $T$ as defined earlier is the vertical distance between tubes centre lines and $D$ is the tube diameter.

For finite cylinders the mean pressure on the cylinder surface is also a direct result of free end interference. Park and Lee [14] reported that the mean pressure on the windward surface increases as we move to the fixed end. This is due to the fact that the vortices shed from two sides of the cylinder become dominant. On the leeward side 
however near the free end the mean pressure is greatly reduced. For the cylinders with both ends fixed, it had been shown that the surface pressure distribution oscillates at the vortex shedding period and that pressure variations are consistent with periodic changes of the circulation around the cylinder body.
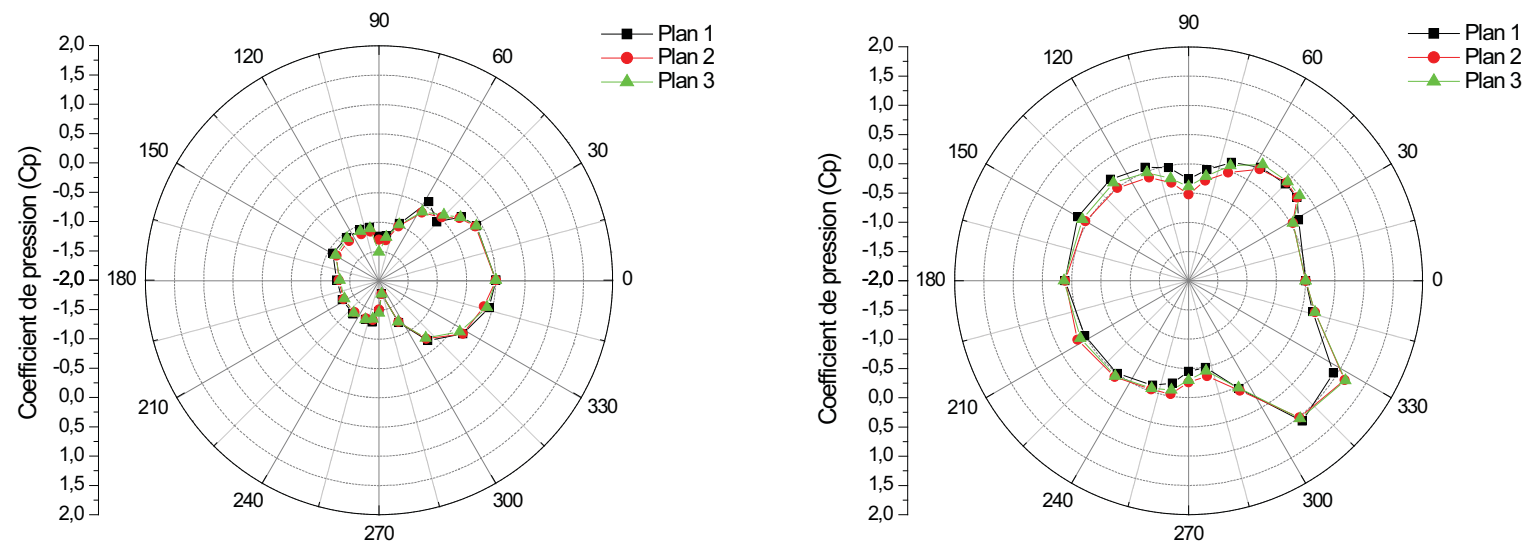

Tube 1-1
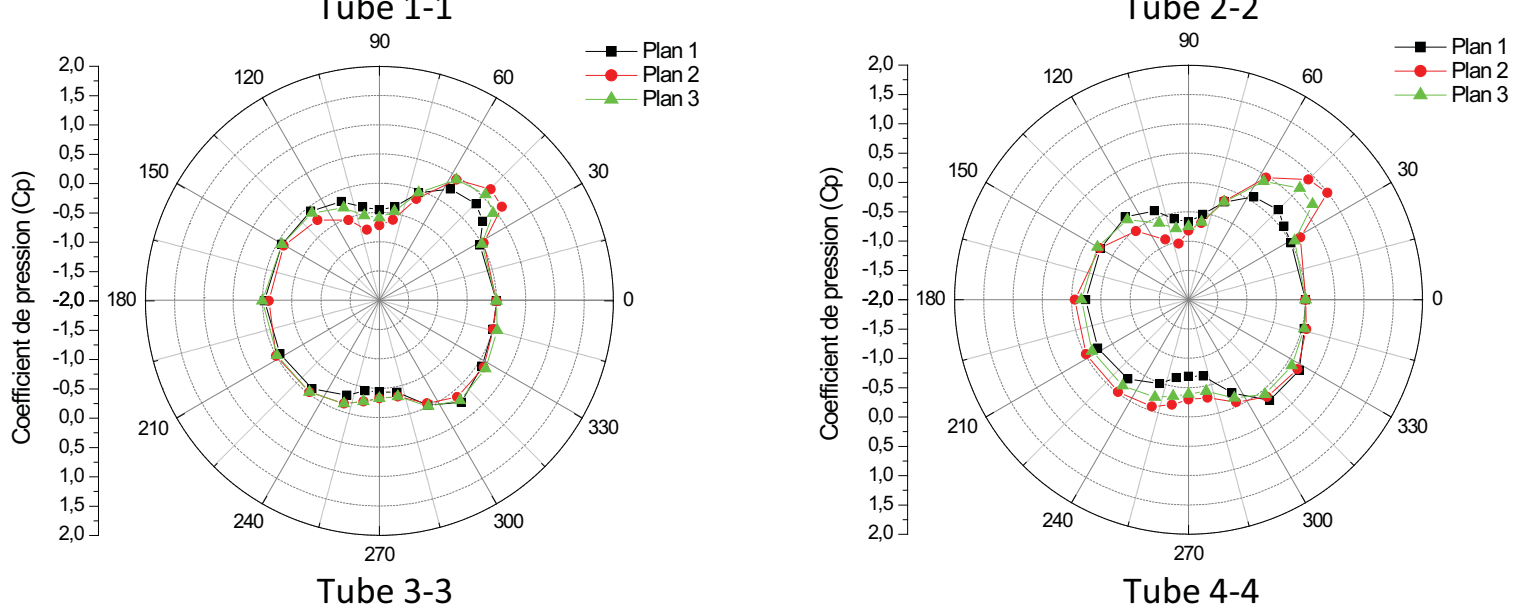

Figure 9: Mean Pressure coefficient distribution for different tubes

Figure 9 represents the mean pressure distribution around the different tube positions (tubes 1-1, 2-2, 3-3, and 4-4). The Plans were respectively situated at $\mathrm{x}=11.5 \mathrm{~cm} ; \mathrm{x}=$ $23 \mathrm{~cm}$ and $x=34.5 \mathrm{~cm}$. It's clearly seen that the stagnation point is located at $0^{\circ}$ for the tube 1-1 and this for each plan but the stagnation point changes angle for the other positions of tubes. For the tubes $2-2$, the curves show that the stagnation point location is around $330^{\circ}$ for all the plans. It has to be noted that for the tubes 3-3 and 4-4, two stagnation points exist, one situated at $45^{\circ}$ and the other at $330^{\circ}$ and this is probably caused by the two contra-rotated vortices on the tube extremities as de-scribed by Aiba et al. [15]. A torsion couple is the result of the location and direction differences between the two vortices. 


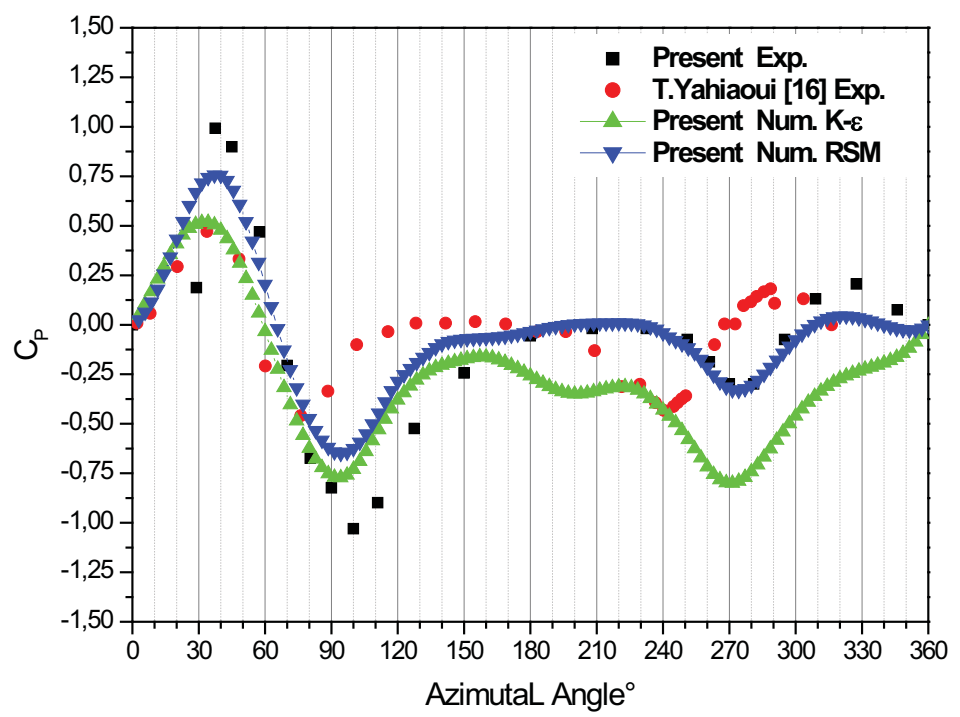

Figure 10: Distribution of normalized pressure coefficient around the central tube (4-4).

Figure 10 shows the evolution of normalized pressure coefficient around the central tube. The effect of flow deflection is observed in term of stagnation pressure region located somewhere around 45 degree from the flow direction which is in good agreement with the experimental results obtained from previous study conducted by Yahiaoui et al. [16]. The minimum pressure for the present experiment is located at around 100 degree because of a separation of a shear layer and a recirculation region and it is delayed compared to the present numerical results where the minimum pressure is located at $90^{\circ}$ degree, the reason of the latter is probably due to the use of grooved cylinders.

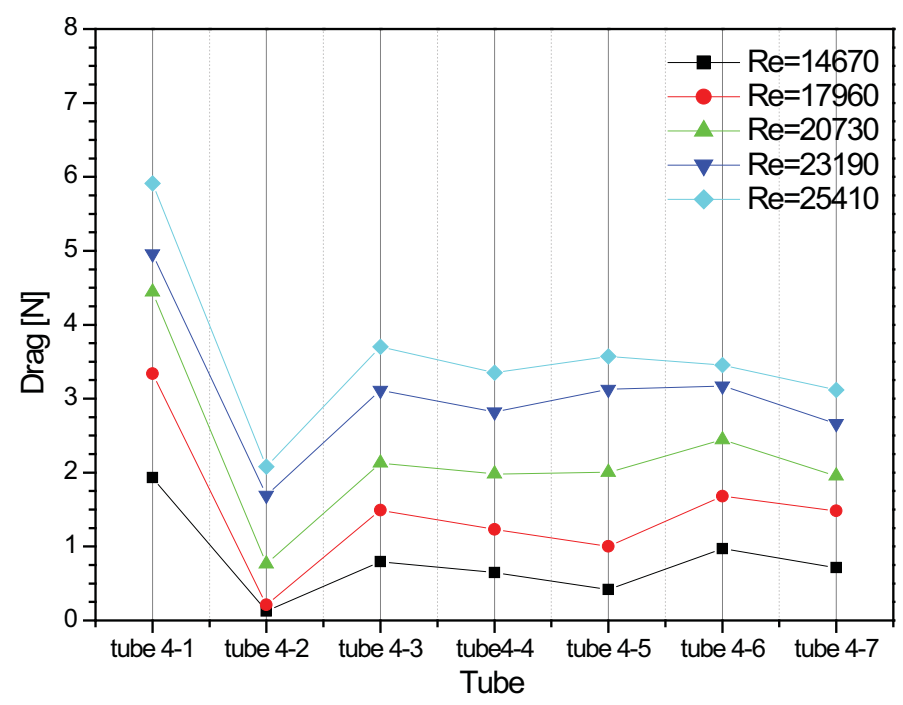

Figure 11: Drag force for different tubes and different Reynolds numbers.

The drag force is presented in figure 11 for different tubes and for various Reynolds number. It is clearly seen that the drag force for the first tube is remarkably higher than the drag for all the other tubes. This finding is valid for all the Reynolds number investigated. It seems that there is a decrease in the drag force for tube 2 for all Reynolds number. 


\section{Conclusion}

The following conclusions have to be drawn from the present work:

- The flow across in-line tube bundles is found to be asymmetric and transient;

- RANS technique in 2D with RSM and approach is able to capture the transient flow behavior, while the $k-\varepsilon$ model suppresses all flow fluctuations.

- Stagnation point is located somewhere around 45 degree. It agrees with Yahiaoui et al. [16] experiment, for the column 3 and 4, two stagnation points exist, one situated at $45^{\circ}$ and the other at $315^{\circ}$.

- Finally, good quantitative and qualitative agreement with the experimental results show the ability of the present approach (RANS) to correctly reproduce the essential physics associated with massively separated flow.

- The drag force for the first tube is remarkably higher than the drag for all the other tubes.

- A decrease in the drag force for tube 2 for all Reynolds number.

\section{REFERENCES}

[1] Sumner, D., Wong, S. S. T., Price, S. J., Paidoussis, M., P. 1999. Fluid behavior of side by side circular cylinders in steady cross-flow. J. Fluids Struct. 13. 309-338.

[2] Kim, H. J., Durbin, P. A. 1988. Investigation of the flow between a pair of circular cylinders in the flopping regime. J. Fluid Mech. 196. 431-448.

[3] Wolfe, D., Zaida, S. 2003. Feedback control of vortex shedding from two tandem cylinders. J. Fluids Struct. 17 579-592.

[4] Feenstra, P. A., Weaver, D. S., Nakamura, T. 2003. Vortex shedding and fluid elastic instability in a normal square tube array excited by two-phase cross-flow. J. Fluids Struct. 17. 793-811.

[5] Paidoussis, M. P. 1982. A review of flow induced vibrations in reactors and reactor components. Nucl. Eng. Design. Vol 74. 31-60.

[6] Weaver, D. S., Fitzpatrick, J. A. 1988. A review of cross flow induced vibrations in heat exchanger tube arrays. J Fluids Struct. 2. 73-93.

[7] Traub, D. 1990. Turbulent heat transfer and pressure drop in plain tube bundles. Chem. Eng. Process. 28. 173-181.

[8] Lam, K., Li, J. Y.,Chan, K. T., So, R. M. C. 2003. Flow pattern and velocity field distribution of cross-flow around four cylinders in a square configuration at a low Reynolds number. J. Fluids Struct. 17. 665-679.

[9] Lam, K., Fang, X. 1995. The effect of interference of four equispaced cylinders in cross flow on pressure and force coefficients. J. FluidsStruct. 9. 195-214.

[10] Benhamadouche, S., Laurence, D. R. 2003. LES, coarse LES, and transient RANS comparisons on the flow across a tube bundle. Int. J. Heat Fluid Flow. 24. 470-479.

[11] Rollet-Miet, P., Laurence, D. R., Ferziger, J. 1999. LES and RANS of turbulent flow in tube bundles. Int. J. Heat Fluid Flow. 20. 241-254.

[12] Moulinec, C., Hunt, J. C. R., Nieuwstadt, F. T. M. 2003. Disappearing wakes and dispersion in numerically simulated flows through tube bundles. Klumwer Academic Publishers. 23-27.

[13] Cheng, Y., Lien, F. S., Yee, E., Sinclair, R. 2003. A comparison of large eddy simulations with a standard k- Reynolds averaged Navier-Stokes model for the prediction of a fully developed turbulent flow over a matrix of cubes. J.Wind Eng. Ind. Aero. 91. 1301-1328.

[14] Park, C.W., Lee, S.J. Free end effect on the wake flow structure behind a finite circular cylinder.-Journal of Wind Eng. and I. Aerodynamics, 2000, 88. p.231-246.

[15] Aiba, S., Tsuchida, H., Ota, T. 1982. Heat transfer around tubes in in-line tube banks. Bull JSME, 25. 919-926; in Flow around circular cylinders Vol 2: Applications by Zdravkovich, M. M.

[16] T. Yahiaoui, L. Adjlout, O. ImineExperimental investigation of in-line tube bundles.MECHANIKA. 2010. Nr.5(85).p 37-43. 